\title{
A Framework for Constructing Features and Models for Intrusion Detection Systems
}

\author{
WENKE LEE \\ Georgia Institute of Technology \\ and \\ SALVATORE J. STOLFO \\ Columbia University
}

Intrusion detection (ID) is an important component of infrastructure protection mechanisms. Intrusion detection systems (IDSs) need to be accurate, adaptive, and extensible. Given these requirements and the complexities of today's network environments, we need a more systematic and automated IDS development process rather than the pure knowledge encoding and engineering approaches. This article describes a novel framework, MADAM ID, for Mining Audit Data for Automated Models for Intrusion Detection. This framework uses data mining algorithms to compute activity patterns from system audit data and extracts predictive features from the patterns. It then applies machine learning algorithms to the audit records that are processed according to the feature definitions to generate intrusion detection rules. Results from the 1998 DARPA Intrusion Detection Evaluation showed that our ID model was one of the best performing of all the participating systems. We also briefly discuss our experience in converting the detection models produced by off-line data mining programs to real-time modules of existing IDSs.

Categories and Subject Descriptors: C.2.0 [Computer-Communication Networks]: General—Security and protection (e.g., firewalls); C.2.3 [Computer-Communication Networks]: Network Operations-Network monitoring; D.4.6 [Operating Systems]: Security and Protection; H.2.8 [Database Management]: Database applications-Data mining; I.2.6 [Artificial Intelligence]: Learning-Concept learning

General Terms: Design, Experimentation, Security

Additional Key Words and Phrases: Data mining, feature construction, intrusion detection

This article is based on the authors' published papers in the Proceedings of the 1999 IEEE Symposium on Security and Privacy and the Proceedings of the 5th ACM SIGKDD International Conference on Knowledge Discovery and Data Mining, as well as Wenke Lee's Ph.D. dissertation in the Computer Science Department at Columbia University.

This research is supported in part by grants from DARPA (F30602-96-1-0311).

Authors' addresses: W. Lee, College of Computing, Georgia Institute of Technology, 801 Atlantic Drive, Atlanta, GA 30332-0280; email: wenke@cc.gatech.edu; S. J. Stolfo, Computer Science Department, Columbia University, 1214 Amsterdam Avenue, Mailcode 0401, New York, NY 10027-7003; email: sal@cs.columbia.edu.

Permission to make digital/hard copy of part or all of this work for personal or classroom use is granted without fee provided that the copies are not made or distributed for profit or commercial advantage, the copyright notice, the title of the publication, and its date appear, and notice is given that copying is by permission of the ACM, Inc. To copy otherwise, to republish, to post on servers, or to redistribute to lists, requires prior specific permission and/or a fee.

(C) 2001 ACM 1094-9224/00/1100-0227 $\$ 5.00$ 


\section{INTRODUCTION}

As network-based computer systems play increasingly vital roles in modern society, they have become the target of intrusions by our enemies and criminals. In addition to intrusion prevention techniques, such as user authentication and authorization, encryption, and defensive programming, intrusion detection is often used as another wall to protect computer systems.

The two main intrusion detection techniques are misuse detection and anomaly detection. Misuse detection systems, for example, IDIOT [Kumar and Spafford 1995] and STAT [Ilgun et al. 1995], use patterns of wellknown attacks or weak spots of the system to match and identify known intrusions. For example, a signature rule for the "guessing password attack" can be "there are more than four failed login attempts within two minutes." Misuse detection techniques in general are not effective against novel attacks that have no matched rules or patterns yet. Anomaly detection (sub)systems, for example, the anomaly detector of IDES [Lunt et al. 1992], flag observed activities that deviate significantly from the established normal usage profiles as anomalies, that is, possible intrusions. For example, the normal profile of a user may contain the averaged frequencies of some system commands used in his or her login sessions. If for a session that is being monitored, the frequencies are significantly lower or higher, then an anomaly alarm will be raised. Anomaly detection techniques can be effective against unknown or novel attacks since no a priori knowledge about specific intrusions is required. However, anomaly detection systems tend to generate more false alarms than misuse detection systems because an anomaly can just be a new normal behavior. Some IDSs, for example, IDES and NIDES [Anderson et al. 1995], use both anomaly and misuse detection techniques.

While accuracy is the essential requirement of an IDS, its extensibility and adaptability are also critical in today's network computing environment. There are multiple "penetration points" for intrusions to take place in a network system. For example, at the network level carefully crafted "malicious" IP packets can crash a victim host; at the host level, vulnerabilities in system software can be exploited to yield an illegal root shell. Since activities at different penetration points are normally recorded in different audit data sources, an IDS often needs to be extended to incorporate additional modules that specialize in certain components (e.g., hosts, subnets, etc.) of the network systems. The large traffic volume in securityrelated mailing lists and Web sites suggests that new system security holes and intrusion methods are continuously being discovered. Therefore IDSs need to be adaptive in such a way that frequent and timely updates are possible.

Currently building an effective IDS is an enormous knowledge engineering task. System builders rely on their intuition and experience to select the statistical measures for anomaly detection [Lunt 1993]. Experts first analyze and categorize attack scenarios and system vulnerabilities, and 
hand-code the corresponding rules and patterns for misuse detection. Because of the manual and ad hoc nature of the development process, current IDSs have limited extensibility and adaptability. Many IDSs only handle one particular audit data source, and their updates are expensive and slow [Allen et al. 2000].

Some of the recent research and commercial IDSs have started to provide built-in mechanisms for customization and extension. For example, both Bro [Paxson 1998] and NFR [Network Flight Recorder Inc. 1997] filter network traffic streams into a series of events, and execute scripts, such as Bro policy scripts and NFR's N-Codes, that contain site-specific event handlers, that is, intrusion detection and handling rules. The system administration personnel at each installation site must now assume the roles of both security experts and IDS builders because they are responsible for writing the correct event-handling functions. Our first-hand experience with both Bro and NFR show that while these systems provide great flexibility, writing the scripts involves a lot of effort, in addition to learning the scripting languages. For example, there is no means to debug the scripts. These systems also handle a fixed set of network traffic event types. On a few occasions we were forced to make changes to the source code of the original IDS to handle new event types.

Our research aims to develop a more systematic and automated approach for building IDSs. We have developed a set of tools that can be applied to a variety of audit data sources to generate intrusion detection models. We take a data-centric point of view and consider intrusion detection as a data analysis process. Anomaly detection is about finding the normal usage patterns from the audit data, whereas misuse detection is about encoding and matching the intrusion patterns using the audit data. The central theme of our approach is to apply data mining programs to the extensively gathered audit data to compute models that accurately capture the actual behavior (i.e., patterns) of intrusions and normal activities. This approach significantly reduces the need to manually analyze and encode intrusion patterns, as well as the guesswork in selecting statistical measures for normal usage profiles. The resultant models can be more effective because they are computed and validated using a large amount of audit data. Furthermore, data mining programs can be applied to multiple streams of evidence, each from a detection module that specializes in a specific type(s) of intrusion or a specific component of the network system (e.g., a missioncritical host) to learn the combined detection model that considers all the available evidence. Therefore, using our framework, IDSs can be extended and adapted easily via automated integration of new modules.

The rest of the article is organized as follows: Section 2 outlines the main components of our framework. Section 3 briefly describes several data mining programs, and discusses how they can be applied to discover frequent intrusion and normal activity patterns, which are the basis for building misuse detection models and user anomaly detection models. Section 4 describes how to construct temporal and statistical features using the frequent patterns mined from audit data. Section 5 reports the results 
of our experiments on building intrusion detection models using the audit data from the DARPA evaluation program. Section 6 briefly describes our approach of converting off-line learned models into real-time intrusion detection modules. Section 7 discusses related research projects. Section 8 outlines our future research plans.

\section{A SYSTEMATIC FRAMEWORK}

A basic premise for intrusion detection is that when audit mechanisms are enabled to record system events, distinct evidence of legitimate activities and intrusions will be manifested in the audit data. Because of the sheer volume of audit data, both in the amount of audit records and in the number of system features (i.e., the fields describing the audit records), efficient and intelligent data analysis tools are required to discover the behavior of system activities.

Data mining generally refers to the process of extracting useful models from large stores of data [Fayyad et al. 1996]. The recent rapid development in data mining has made available a wide variety of algorithms, drawn from the fields of statistics, pattern recognition, machine learning, and databases. Several types of algorithms are particularly useful for mining audit data:

Classification: maps a data item into one of several predefined categories. These algorithms normally output "classifiers," for example, in the form of decision trees or rules. An ideal application in intrusion detection would be to gather sufficient "normal" and "abnormal" audit data for a user or a program, then apply a classification algorithm to learn a classifier that can label or predict new unseen audit data as belonging to the normal class or the abnormal class;

Link analysis: determines relations between fields in the database records. Correlations of system features in audit data, for example, the correlation between command and argument in the shell command history data of a user, can serve as the basis for constructing normal usage profiles. A programmer, for example, may have emacs highly associated with $\mathrm{C}$ files;

Sequence analysis: models sequential patterns. These algorithms can discover what time-based sequence of audit events frequently occur together. These frequent event patterns provide guidelines for incorporating temporal statistical measures into intrusion detection models. For example, patterns from audit data containing network-based denial-ofservice (DoS) attacks suggest that several per-host and per-service measures should be included.

We have developed a framework, MADAM ID (for Mining Audit Data for Automated Models for Intrusion Detection), described in Lee and Stolfo [1998], Lee et al. [1999a; 1999b], and Lee [1999]. The main idea is to apply data mining techniques to build intrusion detection models. The main 


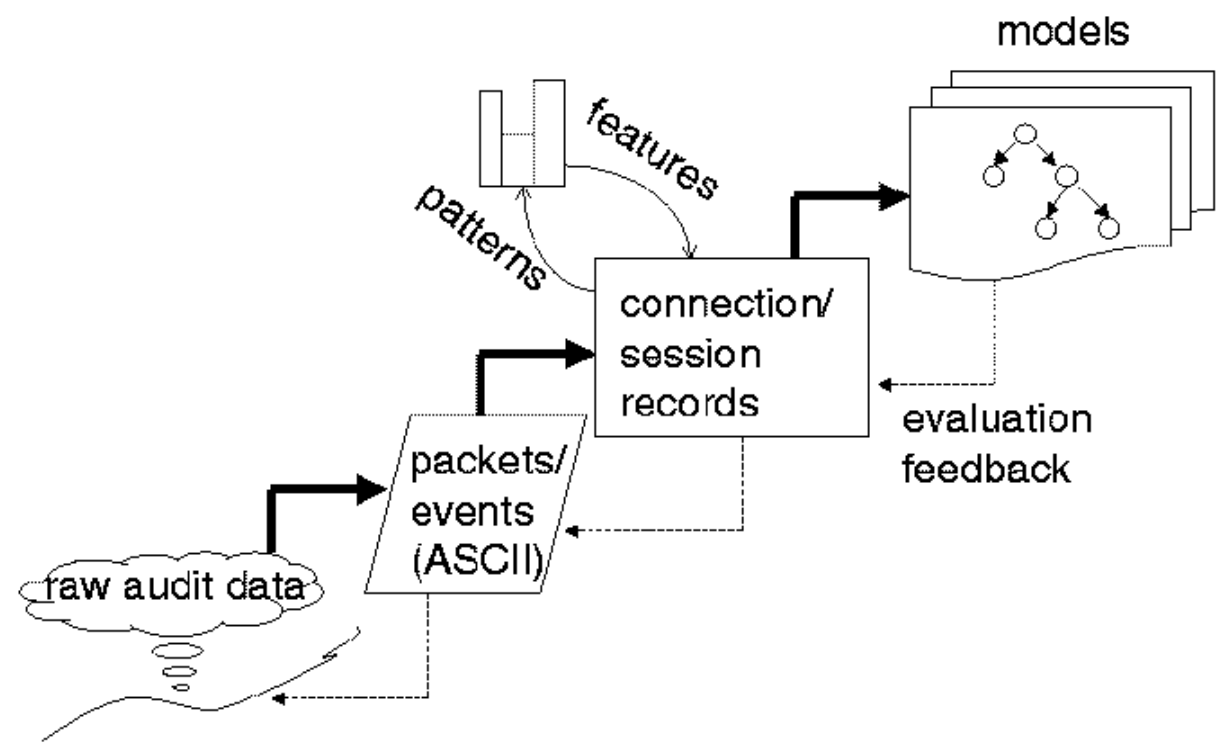

Fig. 1. The data mining process of building ID models.

components of the framework include programs for learning classifiers and meta-classifiers [Chan and Stolfo 1993], association rules [Agrawal et al. 1993] for link analysis, and frequent episodes [Mannila et al. 1995] for sequence analysis. It also contains a support environment that enables system builders to interactively and iteratively drive the process of constructing and evaluating detection models. The end products are concise and intuitive rules that can detect intrusions, and can be easily inspected and edited by security experts when needed.

The process of applying MADAM ID can be summarized in Figure 1. Raw (binary) audit data is first processed into ASCII network packet information (or host event data), which is in turn summarized into connection records (or host session records) containing a number of basic features, such as service, duration, and the like. Data mining programs are then applied to the connection records to compute the frequent patterns (i.e., association rules and frequent episodes), which are in turn analyzed to construct additional features for the connection records. Classification programs, for example, RIPPER [Cohen 1995], are then used to inductively learn the detection models. This process is of course iterative. For example, poor performance of the classification models often indicates that more pattern mining and feature construction is needed.

In our approach, the learned rules replace the manually encoded intrusion patterns and profiles, and system features and measures are selected by considering the statistical patterns computed from the audit data. Meta-learning is used to learn the correlation of intrusion evidence from multiple detection models, and to produce a combined detection model. 
Table I. Telnet Records

\begin{tabular}{|c|c|c|c|c|c|c|c|c|c|}
\hline Label & Service & Flag & hot & failed_logins & compromised & root_shell & $\mathrm{su}$ & Duration & $\ldots$ \\
\hline normal & telnet & $\mathrm{SF}$ & 0 & 0 & 0 & 0 & 0 & 10.2 & $\ldots$ \\
\hline normal & telnet & $\mathrm{SF}$ & 0 & 0 & 0 & 3 & 1 & 2.1 & $\ldots$ \\
\hline guess & telnet & $\mathrm{SF}$ & 0 & 6 & 0 & 0 & 0 & 26.2 & $\ldots$ \\
\hline normal & telnet & $\mathrm{SF}$ & 0 & 0 & 0 & 0 & 0 & 126.2 & $\ldots$ \\
\hline overflow & telnet & $\mathrm{SF}$ & 3 & 0 & 2 & 1 & 0 & 92.5 & $\ldots$ \\
\hline normal & telnet & $\mathrm{SF}$ & 0 & 0 & 0 & 0 & 0 & 2.1 & $\ldots$ \\
\hline guess & telnet & $\mathrm{SF}$ & 0 & 5 & 0 & 0 & 0 & 13.9 & $\ldots$ \\
\hline overflow & telnet & $\mathrm{SF}$ & 3 & 0 & 2 & 1 & 0 & 92.5 & $\ldots$ \\
\hline normal & telnet & $\mathrm{SF}$ & 0 & 0 & 0 & 0 & 0 & 1248 & $\ldots$ \\
\hline$\cdots$ & $\cdots$ & $\cdots$ & $\cdots$ & $\cdots$ & $\cdots$ & $\cdots$ & $\cdots$ & $\cdots$ & $\cdots$ \\
\hline
\end{tabular}

Our framework does not eliminate the need to preprocess and analyze raw audit data, for example, tcpdump [Jacobson et al. 1989] or BSM [SunSoft 1995] audit data. In fact, to build intrusion detection models for network systems, our data mining programs use preprocessed audit data where each record corresponds to a high-level event (e.g., a network connection or host session). Each record normally includes an extensive set of features that describe the characteristics of the event, for example, the duration of a connection, the number of bytes transferred, and so on. While analyzing and summarizing raw audit data is an essential task for an IDS, we argue that generic utilities should first be developed by network and operating system experts, and made available to all IDSs as the low-level building blocks. Bro and NFR can be regarded as examples of such robust utilities, as they both perform IP packet filtering and reassembling, and allow event handlers to output summarized connection records. Our framework assumes that such building blocks are available when constructing IDSs.

Note that currently MADAM ID produces misuse detection models for network and host systems as well as anomaly detection models for users. We are extending MADAM ID to build network and host anomaly detection models. Also note that the detection models produced by MADAM ID are intended for off-line analysis. In Section 6, we briefly discuss how to convert these models to on-line detection modules.

\section{MINING AUDIT DATA}

In this section, we describe our data mining algorithms, and illustrate how to apply these algorithms to generate detection models from audit data. Here audit data refers to preprocessed timestamped audit records, each with a number of features (i.e., fields).

\subsection{Classification}

Intrusion detection can be thought of as a classification problem: we wish to classify each audit record into one of a discrete set of possible categories, normal or a particular kind of intrusion. 
Table II. Example RIPPER Rules from Telnet Records Shown in Table I

\begin{tabular}{|c|c|}
\hline RIPPER Rule & Meaning \\
\hline guess:- failed_logins $\geq 4$. & $\begin{array}{l}\text { If number of failed logins is at least } 4 \text {, then } \\
\text { this telnet connection is "guess", a guessing } \\
\text { password attack. }\end{array}$ \\
\hline $\begin{array}{l}\text { overflow:- hot } \geq 3, \text { compromised } \geq 2 \text {, } \\
\quad \text { root_shell }=1\end{array}$ & $\begin{array}{l}\text { If the number of hot indicators is at least } 3 \text {, } \\
\text { the number of compromised conditions is at } \\
\text { least } 2 \text {, and a root shell is obtained, then this } \\
\text { telnet connection is a buffer overflow attack. }\end{array}$ \\
\hline normal:- true. & $\begin{array}{l}\text { If none of the above, then this connection is } \\
\text { "normal". }\end{array}$ \\
\hline
\end{tabular}

Given a set of records, where one of the features is the class label (i.e., the concept to be learned), classification algorithms can compute a model that uses the most discriminating feature values to describe each concept. For example, consider the telnet connection records shown in Table I. label is the concept to be learned. "normal" represents normal connections and "guess" and "overflow" represent various kinds of intrusions. hot is the count of access to system directories, creation and execution of programs, and so on and compromised is the count of file/path "not found" errors, "Jump to" instructions, and the like. RIPPER [Cohen 1995], a classification rule learning program, generates rules for classifying the telnet connections (see Table II). The symbol to the left of ":-" is the class label, and the comma-separated expressions on the right are conjuncts (i.e., (sub)conditions) of the classification rule. We see that RIPPER indeed selects the discriminating feature values into the classification rules for the intrusions. These rules can be first inspected and edited by security experts, and then be incorporated into misuse detection systems.

The accuracy of a classification model depends directly on the set of features provided in the training data. From a theoretical point of view, the goal of constructing a classification model is that after (selectively) applying a sequence of feature value tests, the dataset can be partitioned into "pure" subsets, that is, each in a target class. Therefore, when constructing a classification model, a classification algorithm searches for features with large information gain [Mitchell 1997], defined as the reduction in entropy, which characterizes the "impurity" of a dataset. It is thus very important that the dataset indeed includes features with large information gain. For example, if the features hot, compromised, and root_shell were removed from the records in Table I, RIPPER would not be able to produce accurate rules to identify buffer overflow connections. In Lee and Stolfo [1998], we showed that due to the temporal nature of network events, especially certain intrusions such as probing (e.g., port-scan, ping-sweep, etc.) and denial-of-service (e.g., ping-of-death, teardrop, etc.), adding per-host and per-service temporal statistics resulted in significant improvement in the accuracy of the classification models. Thus, selecting the right set of system features is a critical step when formulating the classification tasks. Our 
strategy is to first mine the frequent sequential patterns from the network audit data, and then use these patterns as guidelines to select and construct temporal statistical features. Section 3.3 discusses this process in greater detail.

3.1.1 Meta-classification. Meta-learning [Chan and Stolfo 1993] is a mechanism for inductively learning the correlation of predictions by a number of (base) classifiers. Each record in the training data for metalearning contains the true class label of the record and the predictions made by the base classifiers. The resultant meta-classifier thus "combines" the base models because it uses their predictions to make the final prediction. The motivations for meta-learning include: to improve classification accuracy, that is, to produce a meta-classifier that is more accurate than any individual base classifier; and to improve efficiency and scalability, that is, to combine the models rather than the potentially huge volume of data from different data sources. This general approach has been extensively studied [Stolfo et al. 1997] and empirically evaluated in a related domain of credit card fraud detection and has been shown to be effective and scalable.

In order to avoid becoming a performance bottleneck and an easy attack target, an IDS should consist of multiple cooperative lightweight subsystems that each monitors a separate part (e.g., access point) of the entire network environment. For example, an IDS that inspects the full data contents of each IP packet and keeps track of all opened connections may run out of memory (i.e., buffers) during a TCP-based DoS attack and cease to function. On the other hand, a more lightweight IDS that only inspects the header of each IP packet can detect only those intrusions that are aimed at the network protocols, and not those that try to exploit the hosts, such as guess password, buffer overflow, and the like. A solution is to have one relatively lightweight system on the gateway that checks only the packet headers, and several host-based systems that monitor the activities on the mission-critical hosts. A "global" detection system can then combine the evidence from these subsystems and take appropriate actions. We use meta-learning as a means to combine multiple intrusion detection models.

\subsection{Association Rules}

There is empirical evidence that program executions and user activities exhibit frequent correlations among system features. For example, certain privileged programs only access certain system files in specific directories [Ko et al. 1994], programmers edit and compile C files frequently, and so on. These consistent behavior patterns should be included in normal usage profiles.

The goal of mining association rules is to derive multifeature (attribute) correlations from a database table. Given a set of records, where each record is a set of items, support $(X)$ is defined as the percentage of records that contain item set $X$. An association rule is an expression 
Table III. Shell Command Records

\begin{tabular}{ccccc}
\hline Time & Hostname & Command & $\arg 1$ & $\arg 2$ \\
\hline am & pascal & mkdir & $\operatorname{dir} 1$ & \\
am & pascal & cd & $\operatorname{dir} 1$ & \\
am & pascal & vi & tex & \\
am & pascal & tex & vi & \\
am & pascal & mail & fredd & \\
am & pascal & subject & progress & \\
am & pascal & vi & tex & \\
am & pascal & vi & tex & \\
am & pascal & mail & williamf & \\
am & pascal & subject & progress & \\
& $\ldots$ & $\ldots$ & $\ldots$ & $\ldots$ \\
am & pascal & vi & tex & \\
am & pascal & latex & tex & \\
am & pascal & dvips & dvi & - o \\
$\ldots$ & $\ldots$ & $\ldots$ & $\ldots$ & $\ldots$ \\
am & pascal & logout & & \\
\hline
\end{tabular}

$$
X \rightarrow Y,[c, s] .
$$

Here $X$ and $Y$ are item sets, and $X \cap Y=\emptyset, s=\operatorname{support}(X \cup Y)$ is the support of the rule, and $c=\operatorname{support}(X \cup Y) / \operatorname{support}(X)$ is the confidence [Agrawal et al. 1993].

Consider the shell input commands during one telnet session by a secretary, shown in Table III. Here we keep only the filename extensions, remove the (input) contents of mail bodies and files, and use "am" to represent all the morning timestamps.

The original association rules algorithm searches for all possible frequent associations among the set of given features. However, not all associations are necessarily useful for analyzing program or user behavior. We utilized the "schema" level information (i.e., data definitions) about the audit records to direct the pattern mining process. Observe that certain features are essential in describing the data, while others provide only auxiliary information. Domain knowledge is used to determine the appropriate essential features for an application. In shell command data, since the combination of the exact "time" and "command" uniquely identifies each record, "time" and "command" are the essential features; likewise, in network connection data, timestamp, source and destination hosts, source port, and service (i.e., destination port) are the essential features because their combination uniquely identifies a connection record. We argue that the relevant association rules should describe patterns related to the essential features.

We call these essential features(s) axis features when they are used as a form of item constraint, which specifies the conditions on the item sets of an association rule. We restrict the association rules algorithm to only output rules that include axis feature values. In practice, we need not designate all essential features as the axis features. For example, some 
Table IV. Example Association Rules from Shell Command Data Shown in Table III

\begin{tabular}{|c|c|}
\hline Association Rule & Meaning \\
\hline $\begin{array}{l}\text { command }=v i \rightarrow \text { time }=a m, \\
\quad \text { hostname }=\text { pascal, arg } 1=\text { tex, } \\
{[1.0,0.28]}\end{array}$ & $\begin{array}{l}\text { When using vi to edit a file, the user is always } \\
\text { (i.e., } 100 \% \text { of the time) editing a tex file, in the } \\
\text { morning, and at host pascal; and } 28 \% \text { of the } \\
\text { command data matches this pattern. }\end{array}$ \\
\hline $\begin{array}{l}\text { command }=\text { subject } \rightarrow \text { time }=\text { am, } \\
\quad \text { hostname }=\text { pascal, arg } 1= \\
\text { progress },[1.0,0.11]\end{array}$ & $\begin{array}{l}\text { The subject of the user's email is always (i.e., } \\
100 \% \text { of the time) about "progress", such emails } \\
\text { are in the morning, and at host pascal; and } 11 \% \text { of } \\
\text { the command data matches this pattern. }\end{array}$ \\
\hline
\end{tabular}

network analysis tasks require statistics about various network services while others may require the patterns related to the destination hosts. Accordingly, we can use service as the axis feature to compute the association rules that describe the patterns related to the services of the connections, and use destination host as the axis feature to compute patterns related to hosts.

In the case of shell command records, we use command as the axis feature. Table IV shows some example association rules from the shell command data in Table III. Each of these association rules conveys information about the user's behavior. The rules mined from each telnet/ login session of the same user can be merged into an aggregate rule set to form the user's normal profile. Section 5.1.8 details our experiments using association rules for anomaly detection.

\subsection{Frequent Episodes}

There is often the need to study the frequent sequential patterns of audit data in order to understand the temporal and statistical nature of many attacks as well as the normal behavior of users and programs. We use frequent episodes to represent the sequential audit record patterns.

Given a set of timestamped event records, where each record is a set of items, an interval $\left[t_{1}, t_{2}\right]$ is the sequence of event records that starts from timestamp $t_{1}$ and ends at $t_{2}$. The width of the interval is defined as $t_{2}-$ $t_{1}$. Let $X$ be a set of items; an interval is a minimal occurrence of $X$ if it contains $X$ and none of its proper subintervals contains $X$. Define support $(X)$ as the ratio between the number of minimum occurrences that contain $X$ and the total number of event records. A frequent episode rule is the expression [Mannila and Toivonen 1996]

$$
X, Y \rightarrow Z,[c, s, w]
$$

$X, Y$, and $Z$ are item sets, and together they form an episode. $s=$ $\operatorname{support}(X \cup Y \cup Z)$ is the support of the rule, and $c=\operatorname{support}(X \cup Y$ $\cup Z) / \operatorname{support}(X \cup Y)$ is the confidence. The width of each of the occurrences must be less than $w$. 
Table V. Network Connection Records

\begin{tabular}{|c|c|c|c|c|c|c|c|c|}
\hline Timestamp & Duration & Service & src_host & dst_host & src_bytes & dst_bytes & Flag & $\ldots$ \\
\hline 1.1 & 0 & http & spoofed_1 & victim & 0 & 0 & S0 & $\ldots$ \\
\hline 1.1 & 0 & http & spoofed_2 & victim & 0 & 0 & S0 & $\ldots$ \\
\hline 1.1 & 0 & http & spoofed_3 & victim & 0 & 0 & S0 & $\ldots$ \\
\hline 1.1 & 0 & http & spoofed_4 & victim & 0 & 0 & S0 & $\ldots$ \\
\hline 1.1 & 0 & http & spoofed_5 & victim & 0 & 0 & S0 & $\ldots$ \\
\hline 1.1 & 0 & http & spoofed_6 & victim & 0 & 0 & S0 & $\ldots$ \\
\hline 1.1 & 0 & http & spoofed_7 & victim & 0 & 0 & S0 & $\ldots$ \\
\hline$\ldots$ & ... & $\ldots$ & $\ldots$ & $\ldots$ & $\ldots$ & $\ldots$ & $\ldots$ & $\ldots$ \\
\hline 10.1 & 2 & $\mathrm{ftp}$ & $\mathrm{A}$ & $\mathrm{B}$ & 200 & 300 & $\mathrm{SF}$ & $\ldots$ \\
\hline 12.3 & 1 & smtp & $\mathrm{B}$ & $\mathrm{D}$ & 250 & 300 & SF & $\ldots$ \\
\hline 13.4 & 60 & telnet & $\mathrm{A}$ & $\mathrm{D}$ & 200 & 12100 & $\mathrm{SF}$ & $\ldots$ \\
\hline 13.7 & 1 & smtp & B & $\mathrm{C}$ & 200 & 300 & $\mathrm{SF}$ & $\ldots$ \\
\hline 15.2 & 1 & http & $\mathrm{D}$ & $\mathrm{A}$ & 200 & 0 & REJ & $\ldots$ \\
\hline$\ldots$ & $\ldots$ & $\ldots$ & $\ldots$ & $\ldots$ & $\ldots$ & $\ldots$ & $\ldots$ & $\ldots$ \\
\hline
\end{tabular}

We introduced several extensions to the original frequent episodes algorithm. Our extended algorithm computes frequent sequential patterns in two phases: it finds the frequent associations using the axis features(s) as previously described; then it generates the frequent serial patterns from these associations. Thus, our approach combines the associations among features and the sequential patterns among the records into a single rule.

Another interesting schema-level fact about audit records is that some essential features can be the references of other features. These reference features normally carry information about some "subject", and other features describe the "actions" that refer to the same "subject". For example, if we want to study the sequential patterns of connections to the same destination host, then dst_host is the "subject" and service is the action. In this case, we can designate dst_host as the reference feature. When forming an episode, our program tests the condition that, within the episode's minimal occurrences, the event records covered by its constituent item sets have the same reference feature value.

\section{FEATURE CONSTRUCTION}

We use the mined frequent episodes, which also contain associations among the features, from audit records as guidelines to construct temporal statistical features for building classification models. This process involves first identifying the intrusion-only patterns, then parsing these patterns to define features accordingly. In this section, we use network connection data as an example to illustrate the feature construction process.

Raw tcpdump output is first summarized into network connection records using preprocessing programs, where each record has a set of intrinsic features. For example, the duration, service, src_host and dst_host (source and destination hosts), src_port (source port), src_bytes and dst_bytes (number of data bytes), a flag indicating normal or error status according to the protocols, and so on, are intrinsic features of a single connection. Table $\mathrm{V}$ 
Table VI. Example Intrusion Pattern

\begin{tabular}{cl}
\hline Frequent Episode & \multicolumn{1}{c}{ Meaning } \\
\hline flag $=S 0$, service $=$ http, dst_host $=$ & $93 \%$ of the time, after two http connections \\
victim $),($ flag $=S 0$, service $=$ http, & with $S 0$ flag are made to host victim, within \\
dst_host $=$ victim $) \rightarrow$ & 2 seconds from the first of these two, the \\
$($ flag $=S 0$, service $=$ http, dst_host $=$ & third similar connection is made, and this \\
victim $)[0.93,0.03,2]$ & pattern occurs in $3 \%$ of the data \\
\hline
\end{tabular}

shows examples of connection records. Note that these "intrinsic" features are for general network analysis purposes, and not specific to intrusion detection.

\subsection{Identifying the Intrusion Patterns}

We apply the frequent episodes program to both the exhaustively gathered normal connection dataset and the dataset that contains an intrusion. We then compare the resulting patterns to find the intrusion-only patterns, that is, those that exhibit only in the intrusion dataset. The details of the pattern comparison algorithm are described in Lee et al. [1999b]. Briefly, since the number of patterns may be very large and there are rarely exactly matched patterns from two datasets, we used heuristic algorithms to automatically identify the intrusion-only patterns. The idea is to first convert patterns into numbers in such a way that "similar" patterns are mapped to "closer" numbers. Then pattern comparison and intrusion pattern identification are accomplished through comparing the numbers and rank ordering the results. We devised an encoding procedure that converts each pattern into a numerical number, where the order of digit significance corresponds to the order of importance of the features. We used the following heuristic ordering on the importance of the features: flag, the axis feature, the reference feature, the rest of the essential attributes, and then the rest of the features in alphabetical order. flag is considered as the most important in describing a pattern because it carries the summary information of the connection behavior with regard to the protocol specifications. Each unique feature value is mapped to a digit value in the encoding process. The "distance" of two patterns is then simply a number where each digit value is the digit-wise absolute difference between the two encodings. A comparison procedure computes the intrusion score for each pattern from the intrusion dataset, which is its lowest distance score against all patterns from the normal dataset, and outputs the user-specified top percentage patterns that have the highest intrusion scores as the intrusion-only patterns.

As an example, consider the SYN flood attack records shown in Table V. The attacker used many spoofed source addresses to send a lot of S0 connections (i.e., only the first SYN packet is sent) to a port (e.g., http) of the victim host in a very short time span (e.g., all in timestamp 1.1). Table VI shows one of the top intrusion only patterns, produced using service as the axis feature and dst_host as the reference feature. 


\subsection{Constructing Features from Intrusion Patterns}

Each of the intrusion patterns is used as a guideline for adding additional features into the connection records to build better classification models. We use the following automatic procedure for parsing a frequent episode and constructing features:

-Assume $F_{0}$ (e.g., $d s t$ _host) is used as the reference feature, and the width of the episode is $w$ seconds.

-Add the following features that examine only the connections in the past $w$ seconds that share the same value in $F_{0}$ as the current connection:

-A feature that computes "the count of these connections";

-Let $F_{1}$ be service, src_dst, or dst_host other than $F_{0}$ (i.e., $F_{1}$ is an essential feature). If the same $F_{1}$ value (e.g., http) is in all the item sets of the episode, add a feature that computes "the percentage of connections that share the same $F_{1}$ value as the current connection"; otherwise, add a feature that computes "the percentage of different values of $F_{1}$ ";

-Let $V_{2}$ be a value (e.g., S0) of a feature $F_{2}$ (e.g., flag) other than $F_{0}$ and $F_{1}$ (i.e., $V_{2}$ is a value of a nonessential feature). If $V_{2}$ is in all the item sets of the episode, add a feature that computes "the percentage of connections that have the same $V_{2}$ "; otherwise, if $F_{2}$ is a numerical feature, add a feature that computes "the average of the $F_{2}$ values."

This procedure parses a frequent episode and uses three operators, count, percent, and average, to construct statistical features. These features are also temporal since they measure only the connections that are within a time window $w$ and share the same reference feature value. The intuition behind the feature construction algorithm comes from the straightforward interpretation of a frequent episode. For example, if the same feature value appears in all the itemsets of an episode, then there is a large percentage of records that have the same value. We treat the essential and nonessential features differently. The essential features describe the anatomy of an intrusion, for example, "the same service (i.e., port) is targeted." The actual values (e.g., http) are often not important because the same attack method can be applied to different targets (e.g., ftp). On the other hand, the actual nonessential feature values (e.g., flag $=S 0$ ) often indicate the invariant of an intrusion because they summarize the connection behavior according to the network protocols.

This SYN flood pattern shown in Table VI results in the following additional features: a count of connections to the same dst_host in the past 2 seconds, and among these connections, the percentage of those that have the same service, and the percentage of those that have the "S0" flag.

\subsection{Discussions}

We examined the theoretical underpinnings of the feature construction process in Lee [1999]. We outline the results here and explain why the 
features constructed from the intrusion patterns can be utilized to build more accurate classification models. First, the intrusion-only patterns are the results of intrusion records. That is, the "intrusion" dataset must contain "intrusion records," i.e., unique records, unique sequences of records, or records or sequences with unique frequencies, in order for it to have intrusion-only patterns. Second, for the temporal and statistical features constructed from the intrusion patterns, their values in the intrusion records that are responsible for resulting in the intrusion-only patterns will be very different from the feature values in the normal connection records. For example, for the feature from the SYN flood pattern, for the connections to the same destination host in the past 2 seconds, the percentage of those that have $S 0$ flag, normal records have values close to 0 , but SYN flood records have values in the range of greater than $80 \%$. The constructed features have high information gain because their value ranges can separate intrusion records from the normal records. In fact, they normally have higher information gain than the existing set of features. For example, the feature "flag" has very low information gain because some normal connections also have an "S0" value. As discussed in Section 3.1, a classification algorithm needs to select features with the highest information gain when computing a classification model. Therefore, when the features constructed from the intrusion patterns are added to the audit data, a more accurate classification model can be computed. This is precisely the purpose of our feature construction process.

An open problem is how to decide the right time window value $w$. We mine sequential patterns using different $w$ values, for example, from 0.1 to 20 with an increment of 1 , and plot the number of patterns generated at each run. Our experience shows that this plot tends to stabilize after the initial sharp jump. We call the smallest $w$ in the stable region $w_{0}$. In Lee and Stolfo [1998], we reported experiments using different $w$ values to calculate temporal statistical features for classification models. Our results showed the plot of accuracy of the classifier also stabilizes after $w \geq w_{0}$ and tends to taper off. Intuitively, a requirement for a good window size is that its set of sequential patterns is stable; that is, sufficient patterns are captured and noise is small. We therefore use $w_{0}$ for adding temporal statistical features.

\section{EXPERIMENTS}

In this section, we describe our experiments in building intrusion detection models on the audit data from the 1998 DARPA Intrusion Detection Evaluation Program. In these experiments, we applied the algorithms and tools of MADAM ID to process audit data, mine patterns, construct features, and build RIPPER classifiers.

We first describe the experiments on tcpdump data. The results of these experiments were submitted to DARPA and were evaluated by MIT Lincoln Lab. We then report recent experiments on $B S M$ data, which were performed 
after the DARPA evaluation. We discuss our experiences and evaluate the strengths and weaknesses of MADAM ID.

\subsection{Experiments on tcpdump Data}

We participated in the DARPA Intrusion Detection Evaluation Program, prepared and managed by MIT Lincoln Lab [Lippmann et al. 2000]. The objective of this study was to survey and evaluate the state of the art in intrusion detection research. A standard set of extensively gathered audit data, which includes a wide variety of intrusions simulated in a military network environment, was provided by DARPA. Each participating site was required to build intrusion detection models or tweak their existing system parameters using the training data, and send the results (i.e., detected intrusions on the test data) back to DARPA for performance evaluation. We report our experience here.

5.1.1 The DARPA Data. We were provided with about 4 gigabytes of compressed tcpdump data of 7 weeks of network traffic. This data can be processed into about 5 million connection records of about 100 bytes each. The data contains the content (i.e., the data portion) of every packet transmitted between hosts inside and outside a simulated military base. BSM audit data from one UNIX Solaris host for some network sessions were also provided.

The data contains four main categories of attacks:

-DoS, for example, Ping-of-Death, Teardrop, smurf, SYN flood, and so on;

-R2L, unauthorized access from a remote machine, for example, guessing password;

-U2R, unauthorized access to local superuser privileges by a local unprivileged user, for example, various buffer overflow attacks; and

-PROBING, surveillance and probing, for example, Port-Scan, PingSweep, and the like.

In addition, there were anomalous user behaviors such as "a manager becomes (i.e., behaves like) a system administrator."

5.1.2 Data Preprocessing. We used Bro as the packet filtering and reassembling engine. We extended Bro to handle ICMP packets, and made changes to its packet fragment inspection modules since it crashed when processing data that contains Teardrop or Ping-of-Death attacks.

We used a Bro "connection finished" event handler to output a summarized record for each connection. Each connection record included a set of "intrinsic" features shown in Table VII.

5.1.3 Misuse Detection. The training data from DARPA includes "list files" that identify the timestamp, source host and port, destination host and port, and the name of each attack. We used this information to select intrusion data to perform pattern mining and feature construction, and to 
Table VII. Intrinsic Features of Network Connection Records

\begin{tabular}{lll}
\hline Feature & \multicolumn{1}{c}{ Description } & Value Type \\
\hline duration & Length (number of seconds) of the connection & Continuous \\
protocol_type & Type of the protocol, e.g., TCP, UDP, etc. & Discrete \\
service & Network service on the destination, e.g., http, telnet, etc. & Discrete \\
src_bytes & Number of data bytes from source to destination & Continuous \\
dst_bytes & Number of data bytes from destination to source & Continuous \\
flag & Normal or error status of the connection & Discrete \\
land & 1 - connection is from/to the same host/port; 0 - otherwise & Discrete \\
wrong_fragment & Number of "wrong" fragments & Continuous \\
urgent & Number of urgent packets & Continuous \\
\hline
\end{tabular}

label each connection record with "normal" or an attack type to create training data for building classification models.

Since the amount of audit data is huge, for example, some days have several millions of connection records due to some nasty DoS attacks, we did not aggregate all the connection records into a single training data set. Instead, we extracted all the connection records that fell within a surrounding time window of plus and minus 5 minutes of the whole duration of each attack to create a data set for each attack type. We also extracted sequences of normal connection records to create the normal data set that has the same distribution as the original data set.

5.1.4 Manual and Automatic Feature Construction. Following the feature construction approach described in Section 4, for each attack type (e.g., SYN flood, Port-Scan, etc.) we performed pattern mining and comparison using its intrusion data set and the normal data set. We constructed features according to the top $20 \%$ intrusion-only patterns of each attack type. Here we summarize the temporal and statistical features automatically constructed by our system:

- the "same host" features that examines only the connections in the past 2 seconds that have the same destination host as the current connection: - the count of such connections, the percentage of connections that have the same service as the current one, the percentage of different services, the percentage of SYN errors, and the percentage of REJ (i.e., rejected connection) errors;

- the "same service" features that examine only the connections in the past

2 seconds that have the same service as the current connection:

- the count of such connections, the percentage of different destination hosts, the percentage of SYN errors, and the percentage of REJ errors.

We call these the "time-based traffic" features for connection records. They are summarized in Table VIII.

There are several "slow" PROBING attacks that scan the hosts (or ports) using a much larger time interval than 2 seconds, for example, one in every minute or even one in every (few) hour(s). As a result, these attacks did not 
Table VIII. Traffic Features of Network Connection Records

\begin{tabular}{lcc}
\hline Feature & Description & Value Type \\
\hline count & $\begin{array}{l}\text { Number of connections to the same host as the current } \\
\text { connection in the past } 2 \text { seconds } \\
\text { the following features refer to these same-host connections }\end{array}$ & Continuous \\
serror_\% & $\%$ of connections that have "SYN" errors & Continuous \\
rerror_\% & $\%$ of connections that have "REJ" errors & Continuous \\
same_srv_\% & $\%$ of connections to the same service & Continuous \\
diff_srv_\% & $\%$ of connections to different services & Continuous \\
srv_count & Number of connections to the same service as the & Continuous \\
& current connection in the past 2 seconds & \\
srv_serror_\% & the following features refer to these same-service connections & \\
srv_rerror_\% & $\%$ of connections that have "SYN" errors & Continuous \\
srv_diff_host_\% & $\%$ of connections that have "REJ" errors & Continuous \\
\hline
\end{tabular}

produce intrusion-only patterns with the time window of 2 seconds. We sorted the connection records by the destination hosts, and applied the same pattern mining and feature construction process. Instead of using a time window of 2 seconds, we now used a "connection" window of 100 connections, and constructed a mirror set of "host-based traffic" features as the time-based traffic features.

We discovered that unlike most of the DoS and PROBING attacks, the R2L and U2R attacks don't have any intrusion-only frequent patterns. This is because most of the DoS and PROBING attacks involve sending a lot of connections to some host(s) in a very short period of time, and therefore can have frequent sequential patterns that are different from the normal traffic. The R2L and U2R attacks are embedded in the data portions of the packets and normally involve only a single connection. Therefore, it is unlikely that they can have any unique frequent traffic patterns. In other words, our automatic feature construction process, which is based on frequent patterns of connection records, would fail to produce any features for these attacks.

After studying the outcome of this mining process, we focused our attention on the content of the connections. Ideally, we should apply data mining programs to compute patterns from the connection content data and construct appropriate features for R2L and U2R attacks. However, our current data mining algorithms cannot deal with unstructured data contents of IP packets. We instead relied on domain knowledge to define suitable features for R2L and U2R attacks. In the Bro event handlers, we added functions that inspect data exchanges of interactive TCP connections (e.g., telnet, ftp, smtp, etc.). These functions assign values to a set of "content" features to indicate whether the data contents suggest suspicious behavior. These features are: number of failed logins, successfully logged in or not, whether logged in as root, whether a root shell is obtained, whether a $s u$ command is attempted and succeeded, number of access to access control files (e.g., "/etc/passwd", ".rhosts", etc.), number of compromised states on the destination host (e.g., file/path "not found" errors, and "Jump 
Table IX. Content Features of Network Connection Records

\begin{tabular}{|c|c|c|}
\hline Feature & Description & Value Type \\
\hline hot & Number of "hot indicators" & Continuous \\
\hline failed_logins & Number of failed login attempts & Continuous \\
\hline logged_in & 1 - successfully logged in; 0 - otherwise & Discrete \\
\hline compromised & Number of "compromised" conditions & Continuous \\
\hline root_shell & 1 - root shell is obtained; 0 - otherwise & Discrete \\
\hline su & 1 - "su root" command attempted; 0 - otherwise & Discrete \\
\hline file_creations & Number file creation operations & Continuous \\
\hline shells & Number of shell prompts & Continuous \\
\hline access_files & $\begin{array}{l}\text { Number of write, delete, and create operations on access } \\
\text { control files }\end{array}$ & Continuous \\
\hline outbound_cmds & Number of outbound commands in a ftp session & Continuous \\
\hline hot_login & $\begin{array}{l}1 \text { - the login belongs to the "hot" list (e.g., root, adm, etc.); } \\
0 \text { - otherwise }\end{array}$ & Discrete \\
\hline guest_login & $\begin{array}{l}1 \text { - the login is a "guest" login (e.g., guest, anonymous, } \\
\text { etc.); } 0 \text { - otherwise }\end{array}$ & Discrete \\
\hline
\end{tabular}

Table X. Example "Traffic" Connection Records

\begin{tabular}{cccccccc}
\hline Label & Service & Flag & Count & srv_count & rerror_\% & diff_srv_\% & $\ldots$ \\
\hline normal & ecr_i & SF & 1 & 1 & 0 & 1 & $\ldots$ \\
smurf & ecr_i & SF & 350 & 350 & 0 & 0 & $\ldots$ \\
satan & user-level & REJ & 231 & 1 & $85 \%$ & $89 \%$ & $\ldots$ \\
normal & http & SF & 1 & 0 & 0 & 1 & $\ldots$ \\
$\ldots$ & $\ldots$ & $\ldots$ & $\ldots$ & $\ldots$ & $\ldots$ & $\ldots$ & $\ldots$ \\
\hline
\end{tabular}

to" instructions, etc.), number of hot indicators, (e.g., access to system directories, creation and execution of programs, etc.), and number of outbound connections during a ftp session. These features are summarized in Table IX. Our approach here is to include an extensive set of indicators, and then let classification programs decide, from the vast amount of audit data, which minimal set of discriminating features should actually be used to identify intrusions.

5.1.5 Detection Models. It is evident from the feature construction process that different categories of intrusions require different sets of constructed features for detection purposes. We therefore built classification models using different feature sets:

- The "time-based traffic" model: each connection record contains the "intrinsic" and the "time-based traffic" features. Table X shows some example labeled connection records. The resultant RIPPER classifier detects the DoS and PROBING attacks. Table XI shows some example RIPPER rules.

- The "host-based traffic" model: each connection record contains the "intrinsic" and the "host-based traffic" features. The resultant RIPPER classifiers detect the slow PROBING attacks. 
Table XI. Example RIPPER Rules for DoS and PROBING Attacks

\begin{tabular}{|c|c|}
\hline RIPPER Rule & Meaning \\
\hline $\begin{array}{l}\text { smurf:- count } \geq 5, \text { srv_count } \\
\quad \geq 5, \text { service }=\text { ecr_i. }\end{array}$ & $\begin{array}{l}\text { If the service is ICMP echo request, and for the past } 2 \text { seconds, } \\
\text { the number of connections that have the same destination host } \\
\text { as the current one is at least } 5 \text {, and the number of connections } \\
\text { that have the same service as the current one is at least } 5 \text {, } \\
\text { then this is a smurf attack (a DoS attack). }\end{array}$ \\
\hline $\begin{array}{c}\text { satan:- rerror_\% } \geq 83 \%, \\
\text { diff_srv_\% } \geq 87 \% .\end{array}$ & $\begin{array}{l}\text { If for the connections in the past } 2 \text { seconds that have the } \\
\text { same destination host as the current connection, the } \\
\text { percentage of rejected connections is at least } 83 \% \text {, and the } \\
\text { percentage of different services is at least } 87 \% \text {, then this is a } \\
\text { satan attack (a PROBING attack). }\end{array}$ \\
\hline
\end{tabular}

Table XII. Example TCP Connection Records

\begin{tabular}{ccccccccc}
\hline label & service & flag & hot & failed_logins & compromised root_shell & su & $\ldots$ \\
\hline normal & ftp & SF & 0 & 0 & 0 & 0 & 0 & $\ldots$ \\
normal & telnet & SF & 0 & 0 & 0 & 3 & 1 & $\ldots$ \\
guess & telnet & SF & 0 & 6 & 0 & 0 & 0 & $\ldots$ \\
normal & telnet & SF & 0 & 0 & 0 & 0 & 0 & $\cdots$ \\
overflow & telnet & SF & 3 & 0 & 2 & 1 & 0 & $\ldots$ \\
normal & rlogin & SF & 0 & 0 & 0 & 0 & 0 & $\ldots$ \\
guess & telnet & SF & 0 & 5 & 0 & 0 & 0 & $\cdots$ \\
overflow & telnet & SF & 3 & 0 & 2 & 1 & 0 & $\cdots$ \\
normal & telnet & SF & 0 & 0 & 0 & 0 & 0 & $\ldots$ \\
$\ldots$ & $\ldots$ & $\ldots$ & $\ldots$ & $\ldots$ & $\ldots$ & $\ldots$ & $\ldots$ & $\cdots$ \\
\hline
\end{tabular}

-The "content" model: each connection record contains the "intrinsic" and the "content" features. Table XII shows some example labeled connection records. The resultant RIPPER classifier detects the R2L and U2R attacks. Table XIII shows some example RIPPER rules.

These classification models each specializes in a certain type of intrusion. We then constructed a meta-level classifier to combine these detection models. Each meta-level training record consists of four features: the three predictions - each from one of the base models - plus the true class label (i.e., "normal" or an attack type). RIPPER was then applied to learn the rules that combine the evidence from the "time-based traffic,","host-based traffic,"and "content" classifiers to make a (final) prediction on a connection. The resulting meta-level rules basically use the predictions from the "content" model to detect R2L and U2R attacks, and the combination of "time-based traffic" and "host-based traffic" models to detect the DoS and (fast and slow) PROBING attacks. That is, the meta-classifier predicts a connection as an attack of R2L or U2R whenever the "content" model does so; and an attack of DoS or PROBING whenever the "time-based traffic" model does so, or whenever the "time-based traffic" model predicts "normal" but the "host-based traffic" model predicts a PROBING attack.

Table XIV summarizes the complexity of the base models in terms of the number of features in a connection record, the number of RIPPER rules 
Table XIII. Example RIPPER Rules for R2L and U2R Attacks

\begin{tabular}{ll}
\hline \multicolumn{1}{c}{ RIPPER Rule } & \multicolumn{1}{c}{ Meaning } \\
\hline guess:- failed_logins $\geq 4$. & $\begin{array}{l}\text { If number of failed logins is at least 4, then this telnet } \\
\text { connection is "guess", a guessing password attack. }\end{array}$ \\
$\begin{array}{l}\text { If the number of hot indicators is at least 3, the number of } \\
\text { compromised } \geq 2,\end{array}$ & $\begin{array}{l}\text { compromised conditions is at least 2, and a root shell is } \\
\text { obtained, then this telnet connection is a buffer overflow attack. } \\
\text { root_shell }=1 .\end{array}$ \\
$\begin{array}{l}\text {. . . } \\
\text { normal:- true. }\end{array}$ & If none of the above, then this connection is "normal". \\
\hline
\end{tabular}

Table XIV. Model Complexities

\begin{tabular}{cccc}
\hline Model & \#of Features in Records & \#of Rules & \#of Features Used in Rules \\
\hline content & 22 & 55 & 11 \\
traffic & 20 & 26 & $4+\mathbf{9}$ \\
host traffic & 14 & 8 & $1+\mathbf{5}$ \\
\hline
\end{tabular}

produced, and the number of distinct features actually used in the rules. The numbers in bold, for example, $\mathbf{9}$, indicate the number of automatically constructed temporal and statistical features being used in the RIPPER rules. We see that, for both the "traffic" and "host-based traffic" models, our feature construction process contributed the majority of the features. We should point out that not all features in the connection records were selected by RIPPER. This is because RIPPER, like most classification algorithms, has a built-in "feature selection" process to select the most discriminating and generalizable features according to their statistical significance, that is, information gain, and performance on a hold-out test dataset that simulates the "unseen/future" data. Because of the large amount of audit data, a human expert is not able to manually gather and test various statistics, and thus tends to do a poor job in selecting the features. As a result, hand-crafted "signature" rules tend to be very specific to a small intrusion data set. Alternative classification algorithms that compute underlying probability distributions may indeed require all features be evaluated in their resultant models. A crucial issue here is the tradeoff between model accuracy and model cost. The RIPPER output indicates that some features are irrelevant and hence we need not compute these at run-time, thus reducing the cost of detection. This is the subject matter of our ongoing research.

5.1.6 Results. We report the performance of our detection models as evaluated by MIT Lincoln Lab. We trained our intrusion detection models (i.e., the base models and the meta-level classifier) using the 7 weeks of labeled data, and used them to make predictions on the 2 weeks of unlabeled test data (i.e., we were not told which connection was an attack). The test data contained a total of 38 attack types, with 14 types in test data only (i.e., our models were not trained with instances of these attack types; hence, these were considered as "new" attack types). 


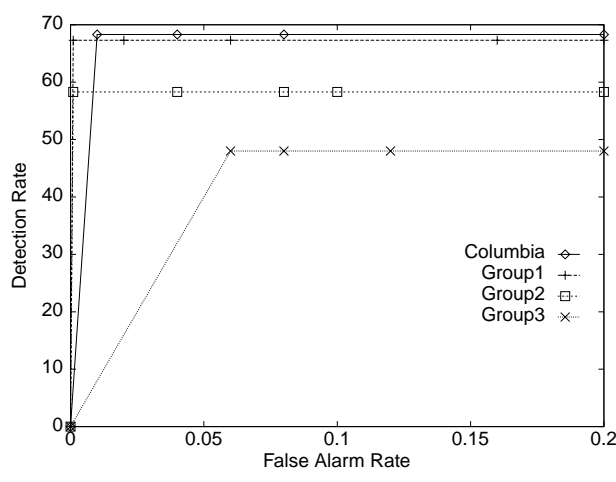

(a) DoS

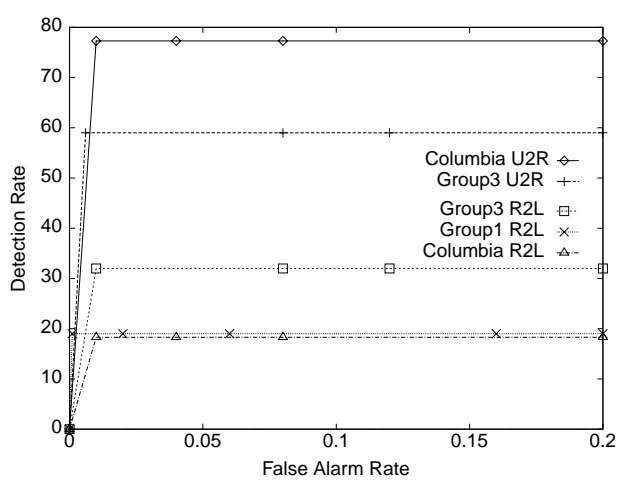

(c) U2R and R2L

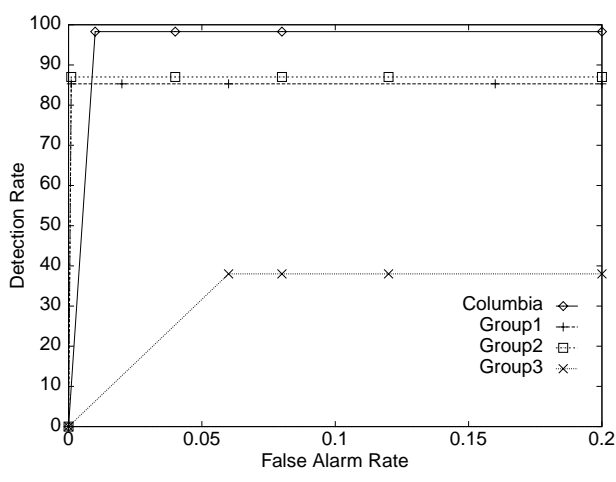

(b) PROBING

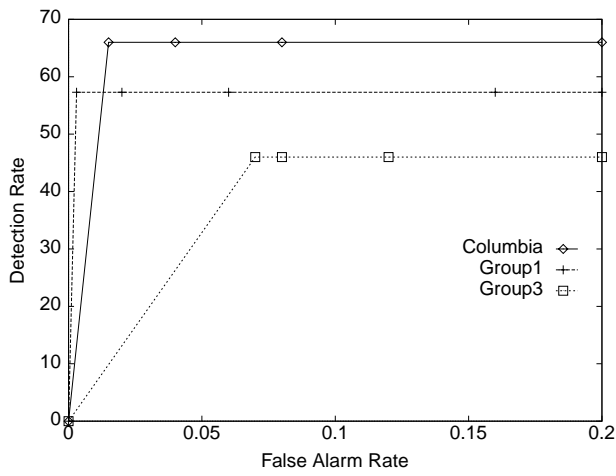

(d) Overall

Fig. 2. Performance of tcpdump misuse detection models: ROC curves on detection rates and false alarm rates.

Figure 2 shows the ROC curves of the detection models by attack categories as well as on all intrusions. In each of these ROC plots, the $\mathrm{x}$-axis is the false alarm rate, calculated as the percentage of normal connections classified as an intrusion; the y-axis is the detection rate, calculated as the percentage of intrusions detected. A data point in the upper left corner corresponds to optimal performance, that is, high detection rate with low false alarm rate. We compare here our models with other participants (denoted as Groups 1 through 3) in the DARPA evaluation program (see the report by Lippmann et. al. [2000]).

Although our models were intended for misuse detection, we had hoped that the features we constructed would be general enough that the models detect new variations of the known intrusions. Table XV compares the detection rates of old intrusions and new intrusions. Here, new intrusions refer to those that did not have corresponding instances in the training data. We see that our models were able to detect a large percentage of new PROBING and U2R attacks, but were not as effective for new DoS and R2L attacks. 
Table XV. Comparing Detection Rates (in \%) on Old and New Attacks

\begin{tabular}{ccr}
\hline Category & Old & New \\
\hline DoS & 79.9 & 24.3 \\
PROBING & 97.0 & 96.7 \\
U2R & 75.0 & 81.8 \\
R2L & 60.0 & 5.9 \\
Overall & 80.2 & 37.7 \\
\hline
\end{tabular}

5.1.7 Discussion. PROBING attacks have relatively limited variance because they all involve making connections to a large number of hosts or ports in a given timeframe. Likewise, the outcome of all U2R attacks is that a root shell is obtained without legitimate means (e.g., login as root, su to root, etc.). Thus, for these two categories of attacks, given some representative instances in the training data, our data mining system was able to construct features that captured their general behavior patterns. As a result, our detection models can detect a high percentage of old and new PROBING and U2R attacks. On the other hand, DoS and R2L have a wide variety of behavior because they exploit the weaknesses of a large number of different network or system services. The features constructed based on the available attack instances were very specialized to the known attack types. Our detection models therefore missed a large number of new DoS and R2L attacks.

The results here are not entirely surprising since our models are misuse detection models. We need to use anomaly detection models on network traffic or system programs to guard against new and diversified attacks. Anomaly detection is much more challenging than misuse detection. For example, we need to first decide whether we should build a normal profile for each network service or group of services, and for each host or group of hosts. The feature construction process will likely be more complex since unlike a relatively small number of intrusion-only patterns, normal network traffic can have a large number of variations. Network anomaly detection is an important problem and an active area of research that we are pursuing.

5.1.8 User Anomaly Detection. Thus far we have discussed only the detection of attacks from outside the network. "Insiders" misusing their privileges can also seriously compromise security. These insider attacks are hard to detect because the insiders don't need to break in. The goal of user anomaly detection is to determine whether the behavior of a user is normal (i.e., legitimate).

It is often very difficult to classify a single event by a user as normal or abnormal because of the unpredictable nature of most people. A user's actions during a login session needs to be studied as a whole to determine whether he or she is behaving normally. We used Bro event handlers to examine the telnet sessions, and extract the shell commands of the users. We further preprocessed the shell commands by replacing timestamps with 
Table XVI. User Descriptions

\begin{tabular}{ll}
\hline User & \multicolumn{1}{c}{ Normal Activities } \\
\hline sysadm & Logs in as root, cats the password file, and runs commands such as top. \\
programmer1 & $\begin{array}{l}\text { Writes public domain C code, uses a vi editor, compiles the C code, reads } \\
\text { and sends mail,and executes UNIX commands. }\end{array}$ \\
programmer2 & A similar user profile, but works in afternoons and evenings. \\
secretary & Edits Latex files, runs Latex, reads and sends mail. \\
manager1 & Reads and sends mail. \\
manager2 & Reads mail. \\
\hline
\end{tabular}

am, pm, and nt (for night), eliminated the input (i.e., contents) of edit and sendmail commands, and kept only the filename extensions. Table III shows examples of the processed command data. These shell command records were used for user anomaly detection.

Our initial exploratory approach was to mine the frequent patterns from the command data, and merge or add the patterns into an aggregate set to form the normal usage profile of a user. A new pattern can be merged with an old pattern if they have the same left- and right-hand sides, their support values are within $5 \%$ of each other, and their confidence values are also within $5 \%$ of each other.

To analyze a user login session, we mine the frequent patterns from the sequence of commands during this session. This new pattern set is compared with the profile pattern set and a similarity score is assigned. Assume that the new set has $n$ patterns and among them, there are $m$ patterns that have "matches" (i.e., rules that they can be merged with) in the profile pattern set; then the similarity score is simply $m / n$. Obviously, a higher similarity score means a higher likelihood that the user's behavior agrees with his or her historical profile.

The DARPA data also included user anomaly data to evaluate anomaly detection systems. Table XVI describes the consistent behavior of the six users for anomaly analysis. Note that since we were the only group that performed anomaly detection on the test data, Lincoln Lab did not evaluate our results. We report our experiments on the training data here.

We applied our frequent episode algorithms to the command data from each login session of the same user, with command as the axis feature and $w=5$ (i.e., we looked for patterns within the range of five consecutive commands), to mine the frequent sequential patterns on the associations among user commands, their arguments, time segments, and hosts. We used the first 4 weeks as a data-gathering period, during which we simply merged the patterns into each user's profiles. Each user has three profiles - one for the activities of each time segment, am, pm, and nt. We used the fifth week as the training period, during which we compared the patterns from each session to the profile of the time segment. We recorded the normal range of the similarity scores during this week. The data in the sixth week had some user anomalies, as described in Table XVII. For each of the anomalous sessions, we compared its patterns against the original 
Table XVII. User Anomaly Description

\begin{tabular}{ll}
\hline User & \multicolumn{1}{c}{ Anomaly Description } \\
\hline programmer2 & Logs in from beta \\
secretary & Logs in at night \\
sysadm & Logs in from jupiter \\
programmer1 & Becomes a secretary \\
secretary & Becomes a manager \\
programmer1 & Logs in at night \\
sysadm & Becomes a programmer \\
manager1 & Becomes a sysadm \\
manager2 & Logs in from pluto \\
\hline
\end{tabular}

user's profile, and then compared the resulting similarity score against the recorded normal range of the same time segment. In Table XVIII, the column labeled "Normal" is the range of similarity of each user against his or her own profile as recorded during the fifth week. A $\infty$ here means that the user did not login during the time segment in the fifth week. The column "Anomaly" is the similarity measure of the anomalous session described in Table XVII. We see that all anomalous sessions can be clearly detected since their similarity scores are much smaller than the normal range. For example, the row in bold in Table XVIII shows that, when the sysadm becomes programmer, his/her patterns have 0 matches with the sysadm's profile; while, for the whole fifth week, the pm similarity scores are in the range of 0.64 to 0.95 .

Once user abnormal behavior is observed, we need to investigate the nature of the anomaly. We report our experiments on finding out how "user job functions" are violated. The problem can be stated as follows: Assume that there are $n$ possible groups of users according to their job functions. When a user in group $i$ does not behave according to the group profile (i.e., the normal job functions), we want to identify which group (e.g., group $j$ ) the user has become. That is, we want to know what "illegal" job functions the user has performed.

In our experiments, we first built group profiles for the $p$ group (the programmers), the $s$ group (the secretary), the $m$ (the managers), and the $s a$ group (the sysadm). From the data of the first 4 weeks, the patterns of all the users of a group were aggregated to form the group profile. The data of the fifth week was used to establish the range of similarity measures for all the users of each group. The user anomalies described in Table XVII include "illegal job function" cases during the sixth week: programmer1 becomes a secretary, secretary becomes a manager, and sysadm becomes a programmer. Table XIX compares the similarity measure of each user in an anomalous session with his/her normal similarity range gathered for the same time segment. From the normal similarity measures of each user with respect to the four groups, we can see that each user indeed has the largest similarity measure with his/her own group. From the similarity measures of the user anomalies, as the bold entries in Table XIX show, for each 
Table XVIII. Similarity Against User's Own Profile: in Normal Use and in the Anomaly Described in Table XVII

\begin{tabular}{llc}
\hline User & \multicolumn{1}{c}{ Normal } & Anomaly \\
\hline programmer2 & $(0.58,0.79)$ & 0.00 \\
secretary & $(\infty, \infty)$ & 0.00 \\
sysadm & $(0.84,0.95)$ & 0.00 \\
programmer1 & $(0.31,1.00)$ & 0.04 \\
secretary & $(0.41,0.98)$ & 0.17 \\
programmer1 & $(\infty, \infty)$ & 0.00 \\
sysadm & $(\mathbf{0 . 6 4}, \mathbf{0 . 9 5})$ & $\mathbf{0 . 0 0}$ \\
manager1 & $(0.57,1.00)$ & 0.00 \\
manager2 & $(1.00,1.00)$ & 0.00 \\
\hline
\end{tabular}

Table XIX. Similarity Against Group Profiles: in Normal Use and in the Anomaly Described in Table XVII

\begin{tabular}{|c|c|c|c|c|c|c|c|c|}
\hline \multirow[b]{2}{*}{ User } & \multicolumn{4}{|c|}{ Normal } & \multicolumn{4}{|c|}{ Anomaly } \\
\hline & $\mathrm{P}$ & $\mathrm{S}$ & M & SA & $\mathrm{P}$ & $\mathrm{S}$ & M & SA \\
\hline p2 & $(0.33,0.71)$ & $(0.04,0.11)$ & $(0.00,0.03)$ & $(0.00,0.07)$ & 0.00 & 0.00 & 0.00 & 0.00 \\
\hline $\mathrm{s}$ & $(\infty, \infty)$ & $(\infty, \infty)$ & $(\infty, \infty)$ & $(\infty, \infty)$ & 0.04 & 0.00 & 0.00 & 0.00 \\
\hline sa & $(0.00,0.00)$ & $(0.00,0.00)$ & $(0.00,0.00)$ & $(0.51,0.81)$ & 0.00 & 0.00 & 0.00 & 0.00 \\
\hline p1 & $(0.12,0.57)$ & $(0.06,0.09)$ & $(0.00,0.00)$ & $(0.04,0.14)$ & 0.04 & 0.11 & 0.00 & 0.00 \\
\hline $\mathbf{s}$ & $(0.02,0.18)$ & $(0.08,0.73)$ & $(0.00,0.00)$ & $(0.00,0.00)$ & 0.17 & 0.00 & 0.50 & 0.00 \\
\hline $\mathrm{p} 1$ & $(\infty, \infty)$ & $(\infty, \infty)$ & $(\infty, \infty)$ & $(\infty, \infty)$ & 0.27 & 0.00 & 0.00 & 0.00 \\
\hline sa & $(0.00,0.00)$ & $(0.00,0.00)$ & $(0.00,0.00)$ & $(0.51,0.81)$ & 0.24 & 0.03 & 0.00 & 0.00 \\
\hline m1 & $(0.14,0.17)$ & $(0.00,0.00)$ & $(0.29,1.00)$ & $(0.00,0.00)$ & 0.02 & 0.00 & 0.00 & 0.61 \\
\hline $\mathrm{m} 2$ & $(0.50,1.00)$ & $(0.00,0.00)$ & $(1.00,1.00)$ & $(0.00,0.00)$ & 0.00 & 0.00 & 0.00 & 0.00 \\
\hline
\end{tabular}

"illegal job function" case, the similarity measure of the targeted group is the largest. For example, when sysadm becomes a programmer, the similarity measure with the $p$ group, 0.24 , is the largest, and the similarity measure with the $s a$ group is outside the normal range.

In summary, although formal evaluation statistics are not available to determine the error rates of our approach in user anomaly detection, the initial results are encouraging. We believe that our approach is worthy of future study.

\subsection{Experiments on BSM Data}

The DARPA data also contains Solaris BSM (Basic Security Module) [SunSoft 1995] audit data for a designated host, pascal. In this section, we describe our experiments in building host-based intrusion detection models using BSM data. The purpose of these experiments was to show that our algorithms for pattern mining and feature construction are not specific to a particular audit data source, for example, tcpdump. We also wanted to investigate whether combining models from tcpdump and BSM can result in better detection performance.

When BSM is enabled in a host machine, there exists an audit trail for the host. An audit trail is a time-ordered sequence of actions that are 
Table XX. Example BSM Event Records

\begin{tabular}{ccccccccc}
\hline Time & auid & sid & event & pid & obname & $\ldots$ & ruid & euid \\
\hline $08: 05: 22$ & 0 & 0 & inetd_connect & 0 & $?$ & $\ldots$ & 0 & 0 \\
$\ldots$ & $\ldots$ & $\ldots$ & $\ldots$ & $\ldots$ & $\ldots$ & $\ldots$ & $\ldots$ & $\ldots$ \\
$08: 05: 22$ & -2 & 0 & execve & 415 & /usr/sbin/in.telnetd & $\ldots$ & 0 & 0 \\
$\ldots$ & $\ldots$ & $\ldots$ & $\ldots$ & $\ldots$ & $\ldots$ & $\ldots$ & $\ldots$ & $\ldots$ \\
$08: 05: 31$ & 2104 & 417 & setaudit & 417 & $?$ & $\ldots$ & 0 & 0 \\
$\ldots$ & $\ldots$ & $\ldots$ & $\ldots$ & $\ldots$ & $\ldots$ & $\ldots$ & $\ldots$ & $\ldots$ \\
$08: 05: 31$ & 2104 & 417 & chdir & 418 & home/tristank & $\ldots$ & 2104 & 2104 \\
$\ldots$ & $\ldots$ & $\ldots$ & $\ldots$ & $\ldots$ & $\ldots$ & $\ldots$ & $\ldots$ & $\ldots$ \\
\hline
\end{tabular}

audited on the system, and consists of one or more audit files. Each audit record in an audit file describes a single audit event, which can be a kernel event (i.e., a system call) or a user-level event (i.e., a system program, such as inetd, in.rshd, etc. invocation).

We define audit session here as the collection of all audit events of an "incoming" or "outgoing" session on the host. Examples of these host sessions include login (e.g., terminal login, telnet login, rlogin, etc.), rsh, $f t p$, sendmail, and so on. It is easy to see that each host session often corresponds to a network connection. We can therefore correlate the predictions on host sessions by a host-based intrusion detection model with the predictions on the corresponding connections by a network intrusion detection model, to yield a higher accuracy.

As in the case of building network intrusion detection models, we also need to first perform a sequence of data preprocessing tasks on the raw BSM data. We extended the preprocessor component of USTAT [Ilgun 1992] to process the binary BSM data into ASCII event data. Table XX shows examples of the event records. Here a "?" means the value is not given in the original BSM audit record. Each event record contains a number of basic features, defined in Table XXI.

We developed a program to process the event data into session records. A brief description of the procedure is the following:

-Watch for the beginning of a session, which is the execution of

- the inetd_connect event (for telnet, rlogin, rsh, etc.), or

- the execve event on a system program in.fingerd (for incoming finger request) or finger (outgoing), mail.local (incoming) or sendmail (outgoing), ftpd (incoming) or ftp (outgoing), and so on.

-Record the setaudit event, which assigns the auid (audit user id) and sid (audit session id) of a session.

-Examine all audit records that share the same combination of auid and sid to summarize a number of session features, which are described in Section 5.2.1.

-Record the termination of a session. 
Table XXI. Features of BSM Event Records

\begin{tabular}{|c|c|c|}
\hline Feature & Description & Value Type \\
\hline time & Timestamp of the event & Discrete \\
\hline auid & $\begin{array}{l}\text { Audit user id, inherited by all child processes started by the } \\
\text { user's initial process of a session }\end{array}$ & Discrete \\
\hline sid & $\begin{array}{l}\text { Audit session id, assigned for each login session and } \\
\text { inherited by all descendant processes }\end{array}$ & Discrete \\
\hline event & Audit event name & Discrete \\
\hline pid & Process id of the event & Discrete \\
\hline obname & $\begin{array}{l}\text { The name of the object, i.e., full path of the file on which } \\
\text { the event operates }\end{array}$ & Discrete \\
\hline $\arg 1-\arg 4$ & Arguments of the system call & Discrete \\
\hline text & Short information of the event, e.g., "successful login" & Discrete \\
\hline error_status & Error status of the event & Discrete \\
\hline return_value & Return value of a system call event & Discrete \\
\hline tmid & Terminal id (port and ip address) of the event & Discrete \\
\hline ip header & $\begin{array}{l}\text { The source and destination ip addresses and ports for the } \\
\text { network connection handled by the event }\end{array}$ & Discrete \\
\hline socket & $\begin{array}{l}\text { The local and remote ip addresses and ports of the socket } \\
\text { used by the event }\end{array}$ & Discrete \\
\hline ruid & The real user id of the event & Discrete \\
\hline rgid & The real group id of the event & Discrete \\
\hline euid & The effective user id of the event & Discrete \\
\hline egid & The effective group id of the event & Discrete \\
\hline
\end{tabular}

The DARPA BSM data contains for each day about 500 sessions on host pascal. The vast majority of the intrusions in the BSM data are U2R buffer overflow attacks.

5.2.1 Defining Session Features. We experimented with feature construction for session records. We first computed frequent patterns from the event records. Here each dataset prepared for pattern mining contains all event records of a session that have positive auid and sid values. That is, we are only interested in events that are "accountable" to the users.

Since we are looking for general rather than session-specific event patterns, we first removed auid and sid from the datasets. We also replaced ruid and euid with a flag same_reid to indicate whether ruid agrees with euid. We designated event as the axis attribute since it is obviously the most essential attribute in describing event data.

After the initial few rounds of experiments, we discovered that the patterns are all related to very specific obname or event values. There are many kernel events (system calls) that cannot be directly linked to userlevel commands. We reasoned that for intrusion detection purposes, we only needed to analyze user-level commands and their operations on the file system. We therefore only kept the following types of event records: read, write, create, delete, execute, change owner or permission, rename, and link. The event value of each event record was replaced by the appropriate type name; for example, open_ $r$ is replaced by read. We only 
kept the original obname if the event was execute; otherwise, we used "user" to replace all obname values that indicated files in the user's directories, and "system" to replace the obname values that indicated files in the system's directories. We also removed all event records that had "?" (i.e., missing) obname values.

We aggregated event patterns of all normal sessions into a normal pattern set. And for each U2R session, we mined its event patterns and compared them with the normal patterns. We used the top $20 \%$ of intrusion-only patterns for each U2R attack, for example,

$$
\begin{aligned}
& (\text { event }=\text { execute }, \text { obname }=/ \text { home/tristank/ffbexploit }, \text { same_reid }=1), \\
& (\text { event }=\text { execute }, \text { obname }=/ \text { usr } / \text { sbin/ffbconfig, same_reid }=0) \rightarrow \\
& (\text { event }=\text { execute }, \text { obname }=/ \text { usr } / \text { bin/ksh }, \text { same_reid }=0)
\end{aligned}
$$

and

$($ event $=$ execute, obname $=/$ usr $/$ bin/pwd, same_reid $=0) \rightarrow$

(event $=$ read, obname $=$ home, same_reid $=0)$.

These patterns are very "unique" because in the normal pattern set, patterns with same_reid $=0$ are those related to read operations only, for example,

$$
\begin{aligned}
& (\text { event }=\text { read }, \text { obname }=\text { system }, \text { same_reid }=0), \\
& (\text { event }=\text { read }, \text { obname }=\text { system }, \text { same_reid }=0) \rightarrow \\
& (\text { event }=\text { read }, \text { obname }=\text { system }, \text { same_reid }=0
\end{aligned}
$$

and

$$
\begin{aligned}
& (\text { event }=\text { read }, \text { obname }=\text { home }, \text { same_reid }=0) \rightarrow \\
& (\text { event }=\text { execute }, \text { obname }=/ \text { usr/bin/cat }, \text { same_reid }=1) .
\end{aligned}
$$

We could have used a mechanical (i.e., automatic) pattern parsing and feature construction procedure for the intrusion-only patterns of the above forms. For example, we could have added features to record the executions of the relevant specific events as described by the patterns. However, we very quickly realized that many U2R buffer overflow attacks share the same characteristics in their intrusion-only patterns. For example, another attack has the following intrusion-only pattern:

$($ event $=$ execute, obname $=/$ home/tristank/formatexploit, same_reid $=1)$,

(event $=$ execute, obname $=/$ usr/bin/fdformat, same_reid $=0) \rightarrow$

$($ event $=$ execute, obname $=/$ usr $/$ bin/ksh, same_reid $=0)$.

These buffer overflow patterns indicate that there is an execution of a user program, follow by a SUID (setuid) system utility, and finally a shell in SIUD state (i.e., a root shell with effective user ID as root and different from the real user ID). We need to construct features that capture the general behavior of the attack method and the final outcome, rather than the specific system utilities being exploited. However, since the event data contains very low-level and specific information of the operating system, we need to use domain knowledge to interpret the patterns to construct the more abstract and general features. Although our experiments here showed the limitations of fully automatic feature construction when dealing with 
Table XXII. Features of BSM Session Records

\begin{tabular}{|c|c|c|}
\hline Feature & Description & Value Type \\
\hline duration & Length (number of seconds) of the session & Continuous \\
\hline service & $\begin{array}{l}\text { Operating system or network service, e.g., telnet, } \\
\text { responsible for this session }\end{array}$ & Discrete \\
\hline logged_in & $\begin{array}{l}\text { Whether the user successfully logged in (when using } \\
\text { telnet, rsh, etc). }\end{array}$ & Discrete \\
\hline failed_logins & Number of failed login attempts & Continuous \\
\hline process_count & Number of processes in the session & Continuous \\
\hline suid_sh & Whether a shell is executed in suid state & Discrete \\
\hline suid_p & Whether a suid system program is executed & Discrete \\
\hline user_p & Whether a user program is executed & Discrete \\
\hline su_attempted & Whether a su command is issued & Discrete \\
\hline access_files & $\begin{array}{l}\text { Number of write, delete, and create operations on } \\
\text { access control files }\end{array}$ & Continuous \\
\hline file_creations & Number of file creations & Continuous \\
\hline hot_login & Whether the login belongs to the "hot" list & Discrete \\
\hline guest_login & Whether the login belongs to the "guest" list & Discrete \\
\hline
\end{tabular}

Table XXIII. Example BSM Session Records

\begin{tabular}{ccccccc}
\hline label & service & suid_sh & suid_p & user_p & file_creations & $\ldots$ \\
\hline normal & smtp & 0 & 0 & 0 & 0 & $\ldots$ \\
normal & telnet & 0 & 1 & 1 & 3 & $\ldots$ \\
normal & telnet & 0 & 1 & 0 & 0 & $\ldots$ \\
buffer_overflow & telnet & 1 & 1 & 1 & 2 & $\ldots$ \\
normal & $\mathrm{ftp}$ & 0 & 0 & 0 & 0 & $\ldots$ \\
wraz_master & $\mathrm{ftp}$ & 0 & 0 & 0 & 42 & $\ldots$ \\
$\ldots$ & $\ldots$ & $\ldots$ & $\ldots$ & $\ldots$ & $\ldots$ & \\
\hline
\end{tabular}

low-level event data, we believe that the intrusion-only patterns resulted from pattern mining and comparison can still provide a very helpful starting point for the manual feature definition process.

We defined a set of features, described in Table XXII, for the BSM session records. Some of the features (i.e., those in bold) are from the buffer overflow patterns, while others are similar to the "content" features described in Section 5.1.3.

5.2.2 Misuse Detection Models on BSM Data. We labeled each BSM session record as "normal" or an intrusion name using the DARPA-supplied list files. BSM session records from all 7 weeks were aggregated into a single dataset for training a misuse detection model. Table XXIII shows some examples of the labeled BSM session records.

RIPPER was applied to the dataset to learn a set of classification rules. Table XXIV shows some examples of these detection rules.

We evaluated the performance of the rule set on the test data using the list files provided by DARPA (available after completion of the official evaluation). Figure 3 shows the ROC curves of the detection models on BSM data. Here Figure 3(a) compares our model with other participants in 
Table XXIV. Example RIPPER Rules for BSM Session Records Shown in Table XXIII

\begin{tabular}{ll}
\hline \multicolumn{1}{c}{ RIPPER Rule } & \multicolumn{1}{c}{ Meaning } \\
\hline buffer_overflow:- suid_sh $=1$ & $\begin{array}{l}\text { If a shell is executed in the SUID state, then this is a buffer } \\
\text { overflow attack. }\end{array}$ \\
wraz_master:- file_creations & $\begin{array}{l}\text { If the service is ftp, the user logs in as a guest (anonymous), } \\
\text { and the number of file creation operations is at least 40, then }\end{array}$ \\
$\geq 40$, service $=$ ftp, & this is a wraz attack (in which the attacker logs to anonymous \\
guest_login $=1$ & $\begin{array}{l}\text { FTP site, creates a hidden directory, and uploads a lot of files, } \\
\text { often pirated copies of software, for others to download). }\end{array}$ \\
&
\end{tabular}

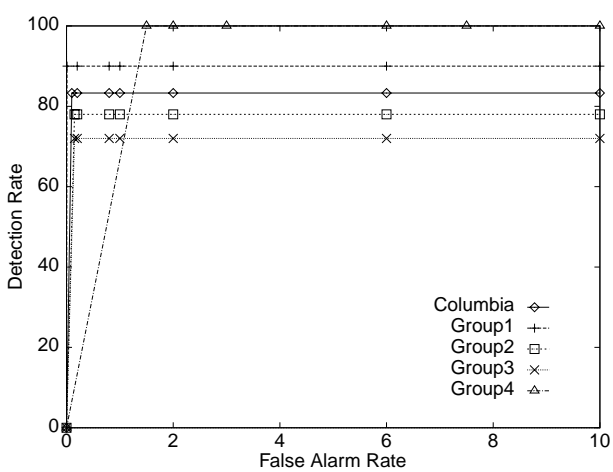

(a) $\mathrm{U} 2 \mathrm{R}$

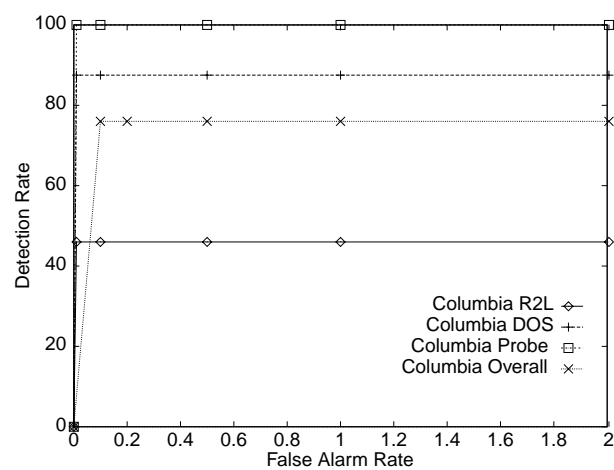

(b) DoS, R2L, PROBING, and overall

Fig. 3. Performance of BSM misuse detection models: ROC curves on detection rates and false alarm rates.

the DARPA Evaluation, in terms of performance in detecting the U2R attacks (DARPA only evaluated performance on U2R). Figure 3(b) shows the performance of our model in detecting DoS, R2L, and PROBING attacks, as well as the overall performance (when all attacks are considered).

The BSM model here may seem to have slightly better performance than the tcpdump models reported in Section 5.1.5. However, the tcpdump models were computed using the tcpdump data of the entire network while the BSM model was constructed using the BSM data, which is available from only one host and contains many fewer attacks and normal sessions. We should note that the BSM model, like the tcpdump models, has good performance in detecting PROBING, U2R, and DoS attacks, but very poor performance in detecting R2L attacks. In fact, when we compared the predictions made by the BSM model and the tcpdump models, we found that they simply agree with each other's predictions on the pairs of corresponding host session and network connection. That is, the BSM misuse detection model, as we have constructed, provides no additional predictive power. This is because:

-by the nature of misuse detection, a truly innovative intrusion, such as one that uses a service that was not modeled, can go undetected, no matter what audit data source is used for analysis; and 
- the features used in the BSM model and the tcpdump models are very similar; that is, the models are looking for similar sets of evidence, although in different data sources.

Although this seems discouraging if we have hoped for an accuracy improvement by combining the two models, this is in fact encouraging if our goal is to combine a lightweight tcpdump model that only checked the IP headers with a number of host-based models that monitor the operating system activities. Our experiments in meta-learning, where a combined model was computed based on a model for tcpdump header-only connection data and a model for BSM host session data, indeed showed that the same level of accuracy was maintained as using a heavyweight tcpdump model that also checked the IP data contents.

\section{CONVERTING LEARNED MODELS TO REAL-TIME IDS MODULES}

Effective intrusion detection should be in real-time to minimize security compromises. We therefore need to study how our models, produced off-line by MADAM ID, can be converted to modules of real-time IDSs, and how they perform in a real-time environment. We are developing a system for translating RIPPER rules into real-time detection modules of NFR (Network Flight Recorder), a system that includes a packet capturing engine and N-code programming support for specifying packet "filtering" logic.

NFR offers a fairly simple framework for network monitoring. It sniffs packets from the network, reassembles them, and then passes them to filter functions for further processing, for example, calculating network traffic statistics. Filter functions are written in $\mathrm{N}$-code, a preemptive, event-driven scripting language that supports a wide range of operations on network packet data. In order to use NFR for network intrusion detection, one needs to implement an extensive set of $\mathrm{N}$-code filters that look for evidence in the network packets.

We seek a mechanical means of converting our off-line detection models into real-time modules. Our approach is to first implement all the required features used in the RIPPER ruleset as N-code "feature filters," and then implement a translator that can automatically convert each RIPPER rule into an N-code "rule filter." For example, a detection rule,

pod:- wrong_fragment $>=1$, protocol_type $=$ icmp.

can be automatically converted into the $\mathrm{N}$-code:

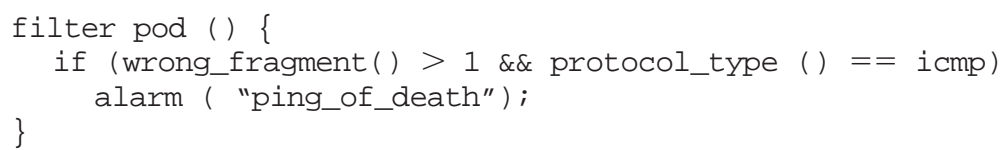

as long as the features (i.e., wrong_fragment and protocol_type) have been implemented as $\mathrm{N}$-code filter functions in NFR.

The advantage of this approach is that when a real-time IDS such as NFR is shipped with MADAM ID to a customer site, MADAM ID can process the local audit data and compute the site-specific intrusion detection rules, which are then automatically converted into N-code "rule filters." 
Although often ignored in off-line analysis, efficiency is a very important consideration in real-time intrusion detection. Specifically, in off-line analysis, it is implicitly assumed that all connections have already finished; therefore, we have the luxury to compute all the features and check the detection rules one by one. Whereas in real-time detection, we need to detect and respond to an intrusion as soon as it happens, often during an ongoing connection. We are therefore studying how to generate an efficient real-time execution plan for a detection ruleset. In particular, we find that many intrusions have cheap "necessary" conditions, which contain features that are easy to compute and are available early in the connection. For example, SYN flood has a necessary condition flag $=S 0$; whereas its sufficient condition includes checking the feature "for the connections to the same destination host as the current connection in the past 2 seconds, the percentage of those that have the S0 flag." The necessary condition is cheaper since the feature flag relies on only the information of the current connection; whereas the feature for the sufficient condition is computed by looking up a number of connection records. Note that a violation of the necessary condition means that there is no need to check the sufficient condition for the intrusion. For example, if flag is not $S 0$, we know that the connection can not be a SYN flood. Therefore, by checking the violations of the cheap necessary conditions first and filtering out a large number of unnecessary checking of ID rules, the overall execution time of the ruleset can be reduced.

We have designed and implemented an algorithm for finding the necessary conditions for intrusions, and are implementing the ruleset filtering algorithm in NFR.

\section{RELATED WORK}

Network intrusion detection has been an ongoing research area [Mukherjee et al. 1994]. More recent systems, for example, Bro [Paxson 1998], NFR [Network Flight Recorder Inc. 1997], and EMERALD [Porras and Neumann 1997] all made extensibility their primary design goals. Both Bro and NFR provide high-level scripting languages for codifying the site-specific intrusion detection rules, which are executed in run-time as event handlers by the packet filtering and reassembly engines. Our research focuses on developing methods for constructing intrusion detection models. Our premise is to use robust IDSs such as Bro and NFR as the building blocks, and provide a framework so that site-specific models can be computed and installed automatically.

EMERALD provides an architecture to facilitate enterprise-wide deployment and configuration of intrusion detectors. The meta-learning mechanism in our framework is designed to automate the process of learning a "resolver," which is needed to combine the alarms from the distributed detectors to make a determination of the state of the (entire) network. The meta-learning results reported in this article are preliminary. We will 
study how to incorporate network configuration information into the metalearning process.

There are several learning-based research efforts in intrusion detection. Warrender et al. [1999] showed that a number of machine-learning approaches (e.g., rule induction, hidden Markov model, etc.) can be used to learn concise and generalizable representation of the "self" identity of a system program, which is the short sequences of run-time system calls made by the program [Forrest et al. 1996]. These learned models were shown to be able to accurately detect anomalies caused by exploits on the system programs. Ghosh and Schwartzbard [1999] showed that, using program activity data (e.g., system calls, arguments, return values, and permissions, etc.) from BSM audit logs, Artificial Neural Networks can be used to learn anomaly and misuse detection models for system programs. Our research aims to develop algorithms and techniques that can be used to build ID models for both networks and hosts. Towards this end, we design the data mining algorithms to be independent of audit data sources. We concentrate our effort on the feature construction process because we believe this is the most critical step in the process of building ID models.

Lane and Brodley developed algorithms for analyzing user shell commands and detecting anomalies [1999]. The basic idea is to first collapse the multicolumn shell commands into a single stream of strings, and then string matching techniques and consideration of "concept drift" are used to build and update user profiles. We believe that our extended frequent episodes algorithm is a superior approach because it considers both the association among commands and arguments, and the frequent sequential patterns of such associations.

\section{CONCLUSIONS AND FUTURE DIRECTIONS}

In this article, we outlined a data mining framework for constructing intrusion detection models. The key idea is to first apply data mining programs to audit data to compute frequent patterns, extract features, and then use classification algorithms to compute detection models. To facilitate adaptability and extensibility, we proposed the use of meta-learning as a means to construct a combined model that incorporates evidence from multiple base models.

We extended the basic association rules and frequent episodes algorithms to accommodate the special requirements in analyzing audit data. Our experiments showed that the frequent patterns mined from audit data can be used as reliable user anomaly detection models, and as guidelines for selecting temporal statistical features to build effective classification models. Results from the 1998 DARPA Intrusion Detection Evaluation Program showed that our detection models performed as well as the best systems built using the manual knowledge engineering approaches.

Anomaly detection models are the only means to detect the truly "innovative" intrusions. Therefore, for future work, we will develop algorithms for learning network anomaly detection models. 
IDSs need to maximize user-defined security goals while minimizing costs. This requires that ID models be sensitive to cost factors, that at the minimum should include the development cost, the operational cost (i.e., the needed resources) the cost of damages of an intrusion, and the cost of detecting and responding to a potential intrusion. For example, when the response cost of an intrusion exceeds its damage cost, the ID models may choose to ignore (i.e., not to respond to) the intrusion. It is particularly important to direct the limited resources (i.e., the computing and human resources) to detect and respond to the most damaging intrusions when the adversaries are launching a large amount of automated attacks in an attempt to overload the IDS. That is, we need ID models that can dynamically determine what are the most cost-saving actions that should be taken. We will extend our framework so that our algorithms can incorporate the user-defined cost factors and policies to compute cost-sensitive ID models.

\section{ACKNOWLEDGMENTS}

We wish to thank our colleagues at Columbia University, Kui Mok, Chris Park, Matt Miller, Wei Fan, and Andreas Prodromidis, for their help and encouragement. We also wish to thank the anonymous reviewers for their helpful comments.

\section{REFERENCES}

Agrawal, R., Imielinski, T., AND Swami, A. 1993. Mining association rules between sets of items in large databases. In Proceedings of the 1993 ACM SIGMOD International Conference on Management of Data (SIGMOD '93, Washington, DC, May 26-28), P. Buneman and S. Jajodia, Eds. ACM Press, New York, NY, 207-216.

Allen, J., Christie, A., Fithen, W., McHugh, J., Pickel, J., And Stoner, E. 2000. State of the practice of intrusion detection technologies. CMU/SEI-99-TR-028,CMU/SEI. Software Engineering Institute, Carnegie Mellon University, Pittsburgh, PA.

Anderson, D., Frivold, T., And Valdes, A. 1995. Next-generation intrusion detection expert system (NIDES): A summary. SRI-CSL-95-07 (May).

Chan, P. K. And Stolfo, S. J. 1993. Toward parallel and distributed learning by metalearning. In Proceedings of the AAAI Workshop on Knowledge Discovery in Databases. $227-240$.

Cohen, W. W. 1995. Fast effective rule induction. In Proceedings of 12th International Conference on Machine Learning (Lake Tahoe, CA). Morgan Kaufmann, San Mateo, CA.

FAYYAD, U., PiAtetsky-Shapiro, G., AND Smyth, P. 1996. The KDD process of extracting useful knowledge from volumes of data. Commun. ACM 39, 11, 27-34.

Forrest, S., Hofmeyr, S. A., Somayaji, A., And Longstaff, T. A. 1996. A sense of self for Unix processes. In Proceedings of the IEEE Symposium on Security and Privacy (Oakland, CA, May). IEEE Press, Piscataway, NJ, 120-128.

Ghosh, A. K. And SchwartzBard, A. 1999. A study in using neural networks for anomaly and misuse detection. In Proceedings of the 8th Security Symposium on USENIX (USENIX, Aug.).

ILGUN, K. 1992. USTAT: A real-time intrusion detection system for Unix. Master's Thesis. University of California at Santa Barbara, Santa Barbara, CA.

Ilgun, K., Kemmerer, R. A., AND Porras, P. A. 1995. State transition analysis: A rule-based intrusion detection approach. IEEE Trans. Softw. Eng. 21, 3 (Mar.), 181-199.

Jacobson, V., Leres, C., And McCanne, S. 1989. Tcpdump. available via anonymous ftp to ftp.ee.lbl.gov. 
Ko, C., Fink, G., AND LevitT, K. 1994. Automated detection of vulnerabilities in privileged programs by execution monitoring. In Proceedings of the 10th Conference on Computer Security Applications (Dec.). IEEE Computer Society Press, Los Alamitos, CA, 134-144.

Kumar, S. AND Spafford, E. H. 1995. A software architecture to support misuse intrusion detection. In Proceedings of the 18th National Conference on Information Security. 194-204.

Lane, T. And Brodley, C. E. 1999. Temporal sequence learning and data reduction for anomaly detection. ACM Trans. Inf. Syst. Secur. 2, 3, 295-331.

LEE, W. 1999. A data mining framework for constructing features and models for intrusion detection systems. Ph.D. Dissertation. Columbia University, New York, NY.

Lee, W. AND Stolfo, S. J. 1998. Data mining approaches for intrusion detection. In Proceedings of the 7th Symposium on USENIX Security (San Antonio, TX, Jan.).

LeE, W., Stolfo, S. J., AND MoK, K. W. 1999. A data mining framework for building intrusion detection models. In Proceedings of the 1999 IEEE Symposium on Security and Privacy (Oakland, California, May).

Lee, W., Stolfo, S. J., ANd MoK, K. W. 1999. Mining in a data-flow environment: Experience in network intrusion detection. In Proceedings of the 5th ACM SIGKDD International Conference on Knowledge Discovery \& Data Mining (KDD-99).

Lippmann, R. P., Fried, D., Graf, I., Haines, J., Kendall, K., McClung, D., Webber, D., Webster, S., Wyschograd, D., Cunninghan, R., And Zissman, M. 2000. Evaluating intrusion detection systems: The 1998 DARPA off-line intrusion detection evaluation. In Proceedings of the on DARPA Information Survivability Conference and Exposition (DISCEX '00, Hilton Head, South Carolina, Jan. 25-27). IEEE Computer Society Press, Los Alamitos, CA, 12-26.

Lunt, T. 1993. Detecting intruders in computer systems. In Proceedings of the 1993 Conference on Auditing and Computer Technology.

Lunt, T., Tamaru, A., Gilham, F., Jagannathan, R., Neumann, P., Javitz, H., Valdes, A., And GARVEY, T. 1992. A real-time intrusion detection expert system (IDES) - final technical report.

Mannila, H. And Toivonen, H. 1996. Discovering generalized episodes using minimal occurrences. In Proceedings of the 2nd International Conference on Knowledge Discovery in Databases and Data Mining (Portland, OR, Aug.).

Mannila, H., Toivonen, H., AND Verkamo, A. I. 1995. Discovering frequent episodes in sequences. In Proceedings of the First International Conference on Knowledge Discovery in Databases and Data Mining (Montreal, Canada, Aug. 20-21).

Mitchell, T. 1997. Machine Learning. McGraw-Hill, Inc., New York, NY.

MukherJee, B., Heberlein, L. T., AND LevitT, K. N. 1994. Network intrusion detection. IEEE Network 8, 1 (Jan.).

Network Flight ReCORder INC. 1997. Network flight recorder. http://www.nfr.com

PAXson, V. 1998. Bro: A system for detecting network intruders in real-time. In Proceedings of the 7th Symposium on USENIX Security (San Antonio, TX, Jan.).

Porras, P. AND NEumann, P. 1997. EMERALD: Event monitoring enabling responses to anomalous live disturbances. In Proceedings of the 20th National Conference on National Information Systems Security. Vol.1 (Baltimore, MD). National Institute of Standards and Technology, Gaithersburg, MD, 353-365.

Stolfo, S. J., Prodromidis, A. L., Tselepis, S., Lee, W., Fan, D. W., And Chan, P. K. 1997. JAM: Java agents for meta-learning over distributed databases. In Proceedings of the 3rd ACM SIGMOD International Workshop on Data Mining and Knowledge Discovery (SIGMOD-96, Newport Beach, CA, Aug.), R. Ng, Ed. ACM Press, New York, NY, 74-81.

SunSoft. 1995. SunSHIELD Basic Security Module Guide.

Warrender, C., Forrest, S., AND Perlmutter, B. 1999. Detecting intrusions using system calls: Alternative data models. In Proceedings of the 1999 IEEE Computer Society Symposium on Research in Security and Privacy (Berkeley, CA, May). IEEE Computer Society Press, Los Alamitos, CA, 133-145.

Received: February 2000; revised: October 2000; accepted: November 2000 